\title{
Abstracts of the meeting of the Clinical Genetics Society held on 18 and 19 November 1983 at Guy's Hospital, London
}

\author{
X linked dominant chondrodysplasia punctata: a case \\ report and family studies \\ R F MUELLER*, R A K JONES $\dagger$, P M CROWLE $\ddagger$, AND B C C \\ DAVISON* \\ *Department of Genetic Counselling, and $\dagger$ Department of \\ Paediatrics, Addenbrooke's Hospital, Cambridge; and \\ $\ddagger$ Department of Paediatrics, Norfolk and Norwich \\ Hospital, Norwich.
}

Chondrodysplasia punctata is a syndrome with cutaneous, ocular, and skeletal involvement. Two major genetic types have been delineated; a severe rhizomelic form usually lethal in the first year of life which is recessively inherited, and the less severe Conradi-Hünermann form which appears to be dominantly inherited. Clinicogenetic analyses of patients with this latter form of the disease has revealed a sub-group characterised by asymmetrical involvement with linear or whorled patches of ichthyosiform erythroderma or atrophoderma of the skin, unilateral cataracts, and asymmetrical limb shortening. The limitation of reported patients to females and the mosaic pattern of the clinical features consistent with Lyonisation are suggestive of an $X$ linked dominant gene which is lethal in the hemizygous male. Four instances of familial occurrence of this form of chondrodysplasia punctata have been reported. We reported a $2 \frac{1}{2}$ year old girl with ichthyosiform skin patches, circumscribed alopecia, coarse lustreless hair, unilateral right sided cataract, and asymmetrical shortening of the left arm and leg. Neonatal skeletal roentgenograms revealed widespread epiphyseal stippling. The child's mother and her three sisters all have symmetrical short stature and patches of ichthyosiform atrophy. The maternal grandmother, in addition, has sparse hair, scoliosis, and a unilateral short arm. There are no male offspring for three generations and no history of early miscarriages. Discussion included a review of the clinical features of the family and the previously reported family studies supporting this mode of inheritance of chondrodysplasia punctata.

The HARD $\pm E$ syndrome

PETER FARNDON AND DIAN DONNAI

Department of Medical Genetics, St Mary's Hospital, Hathersage Road, Manchester M13 OJH.

The majority of cases of hydrocephalus occur either in association with spina bifida or as a single malformation with no other abnormalities. Most do not have a pattern of inheritance suggestive of a single gene defect. However, infants with hydrocephalus associated with agyria, $\vec{\overrightarrow{ }}$ retinal dysplasia with or without an encephalocele form $\vec{\omega}$ a distinct sub-group (the HARD $\pm E$ syndrome) and have been shown to have a high recurrence risk. We reported two families (one consanguineous) who had male and iv female sibs with the HARD $\pm E$ syndrome giving further $\vec{\sim}$ support to an autosomal recessive mode of inheritance. ? The clinical and pathological findings were discussed. $\vec{\omega}_{\infty}$ These confirm the variation in signs of this syndrome between sibs. Because of the genetic implications of the $H A R D \pm E$ syndrome, careful external examination and detailed macroscopic and microscopic pathological $\frac{D}{O}$ investigations are indicated in infants who die with hydrocephalus. Joint hypermobility syndrome: an inherited collagen
disorder?

A CHILD, D SYMMONS, N LIGHT, M DYSON, D DORRANCE, ٌํำ AND R GRAHAME

Departments of Rheumatology and Anatomy, Guy's Hospital, and ARC Meat Research Institute, Bristol.

Previous studies have confirmed the association between articular symptoms, hypermobility, and mitral valve prolapse, and the hereditary nature of ligamentous laxity. In a group of 72 hypermobility patients $(62 \mathrm{~F}: 10 \mathrm{M})$ and their immediate families, physical examination for signs $\dot{0}$ of connective tissue weakness was accompanied by measurement of aortic compliance by non-invasive $\hat{\rho}$ continuous wave doppler ultrasound (normal values 3 already established in over 800 volunteers of both sexes). Aortic compliance (AC) was raised above +2 SD in 44 근 of 72 probands, indicating decreased strength of the $\frac{D}{O}$ aortic wall, and providing a non-invasive screening procedure. A positive family history of joint hyper- $N$ mobility and/or early onset arthralgia was obtained from or 53 of 72 probands (73\%). Two generation involvement $\mathrm{N}$ was demonstrated by raised AC levels in affected family members of 17 of 20 probands. A sex influenced dominant $\sigma$ mode of inheritance is most likely, with affected females having higher hypermobility scores and being more

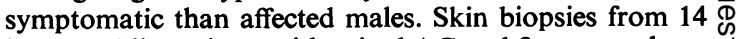
hypermobile patients with raised $\mathrm{AC}$ and five normal age ${ }^{+}$ and sex matched controls revealed significantly decreased $T$ type III collagen in 5 of 14 patients. Electron microscopy revealed markedly decreased collagen fibrils. This study suggests a biochemical basis for genetic heterogeneity and $\mathbb{D}$ raises the possibility of specific therapy through modi- $\frac{\sigma}{\sigma}$ fication of collagen production. 


\section{Congenital heart defects and twinning}

JOHN BURN

Clinical Genetics Unit, Institute of Child Health, 30 Guilford Street, London WCIN IEH.

In 1961 Campbell suggested that monozygotic twinning may be associated with an excess of cardiac malformations. Subsequent studies have been compatible with this hypothesis, but none to date has combined adequate proof of zygosity with unbiased ascertainment and precise cardiac diagnoses in a sufficiently large series to provide confirmation. Over 330 families in which a child resulting from a multiple pregnancy had a cardiovascular malformation have been ascertained in five centres; through the records of Freeman Hospital, Newcastle, and The Hospital for Sick Children, Great Ormond Street, London, and from the twin/malformation registries in Aberdeen, Birmingham, and Liverpool. Where possible twin pairs have been examined and zygosity confirmed by examination of blood polymorphisms. Even after excluding conjoined twins and persistent ductus arteriosus, there is a significant excess of heart defects among monozygotic twins. It is likely that the twinning process itself affects heart development in one of the pair. It was proposed that disturbance of laterality might be an important mechanism. The twin study method cannot easily be applied to cardiac malformations; previous attempts to do so have resulted in an underestimate of the contribution of genetic factors to heart malformations in singletons.

\section{Prediction counselling for Huntington's chorea PETER S HARPER AND AUDREY TYLER Welsh National School of Medicine, Cardiff.}

Despite widespread discussion of the problems likely to be associated with a predictive test for Huntington's chorea, the absence of any reliable test has precluded a clear plan of action. Recent developments in molecular genetics and gene mapping (Gusella et al. Nature 1983; 306:234) have identified a closely linked DNA polymorphism that may be applicable as a predictive marker so that preparation of families is now essential. Three phases of counselling for persons at risk are planned, each with a specific protocol. (1) Preparation for a predictive test, including explanation of the limitations of the testing and assessment of the stability of the person. (2) Immediate post-test counselling, particularly in relation to support of those found likely to carry the gene. (3) Follow up counselling and assessment of probable gene carriers. Problems are anticipated whatever approach is used, but we consider that a combination of careful explanation, support, and assessment is likely to minimise them.

\section{Autosomal or sex linked: a counselling dilemma \\ I D YOUNG AND $Z$ NUGENT \\ Department of Child Health, University of Leicester, Leicester.}

When advice is sought by the sister of two or more brothers, each of whom has an identical but undiagnosed or 'new' syndrome, it is vital to try to derive the relative probabilities of autosomal recessive and sex linked recessive inheritance. The approach to these calculations is difficult and receives scant attention in genetics texts. It is proposed that standard Bayesian analysis may be applied in this situation. For a MCA/MR syndrome, prior odds of 19:1 based on the relative length of the $\mathrm{X}$ chromosome are appropriate. The conditional odds should take into account the absence of consanguinity and the constitution of the family pedigree, including the number of maternal uncles. In the special instance of non-specific mental retardation empirical data indicate that genes on the $\mathrm{X}$ chromosome have a disproportionate impact and that prior odds of approximately 1:2 may be used for autosomal recessive:sex-linked recessive inheritance (Herbst DS, Miller JR. Am J Med Genet 1980;7: 461-9).

A study of the incidence and heterogeneity of retinitis pigmentosa within the city of Birmingham $S$ BUNDEY AND $S$ J CREWS

Birmingham and Midland Eye Hospital, Birmingham.

An attempt was made to ascertain all symptomatic cases of retinitis pigmentosa (RP) living within the city of Birmingham in June 1978. A prevalence of 1 in 3000 for persons aged 50 to 60 years was obtained, and there are reasons for thinking that this may be an underestimate. The 215 patients were then classified according to the mode of inheritance of their disease, except for $8 \%$ who were not further available. Recognisable autosomal dominant inheritance accounted for $30 \%$ of patients in the city, autosomal recessive inheritance for only about $7 \%$, X linked recessive inheritance for $17 \%$, and autosomal recessive syndromes for $14 \%$. Penetrance of autosomal dominant RP was low below the age of 40 years. In contrast, all obligatory heterozygotes for $X$ linked RP could be identified over the age of 20 . Twenty-four percent of cases were isolated and could not be explained by chance-isolated recessive inheritance. These isolated cases were likely either to have autosomal dominant disease (with asymptomatic relatives) or to have a non-genetic disorder.

Achromatopsia and combination defects of protan, deutan, and tritan genes

L N WENT AND N PRONK

Department of Human Genetics, University of Leiden, The Netherlands.

The studies reported here originate from five index cases with 'achromatopsia'. In all instances family members were studied for the presence of inherited colour vision defects. In one family with $\mathrm{X}$ chromosomal achromatopsia with typical achromatic brightness matches on the Nagel anomaloscope, it was tentatively concluded that the achromatopsia is caused by the presence of genes for protanopia and deuteranopia on a single $\mathrm{X}$ chromosome. It was also concluded that the genes for protan and 
deutan defects are very close to each other on the $\mathrm{X}$ chromosome, making a recombinational event between the two extremely rare. A man with achromatopsia and normal visual acuity had a protanopic brightness match on the anomaloscope. His father and other paternal relatives had tritan defects, leading to the conclusion that this achromatopsia is caused by the simultaneous presence of genes for $\mathbf{X}$ linked protanopia and for an autosomal tritan defect; thus only green sensitive cones would remain. Achromatopsia in a female with normal visual acuity is interpreted in the same way by stipulating two genes for $\mathrm{X}$ linked deutan defects and a gene for a tritan defect. A family with a mother and six of 10 children affected with achromatopsia, low visual acuity, photophobia, and nystagmus can only be explained by the presence of pseudodominance of a gene for autosomal recessive achromatopsia.

A genetic linkage study of $X$ linked retinitis pigmentosa A F WRIGHT, S S BHATTACHARYA, W H PRICE, A D CAROTHERS, J F CLAYTON, C M E MCKEOWN, C I PHILLIPS, M JAY, A C BIRD, E M SOUTHERN, AND H J EVANS

MRC Clinical and Population Cytogenetics Unit, Western General Hospital, Edinburgh.

The $\mathrm{X}$ linked variety of retinitis pigmentosa (XLRP) is one of the most severe clinical forms of this disorder and there is a need to develop accurate means of genetic counselling. A recombinant DNA library has been prepared from flow sorted human $\mathbf{X}$ chromosomes and clones containing $X$ chromosome specific unique sequences have been selected. One of these clones (p212) and two clones donated by other laboratories have teen used to try to locate the gene(s) for XLRP using linkage analysis. DNA from 30 obligate XLRP heterozygotes have been screened using RC8 (Davies, London), L1.28 (Pearson, Leiden), and p212, each of which identifies a restriction fragment length polymorphism (RFLP) with TaqI. Nine obligate heterozygotes were informative for L1.28 and eight for p212. The segregation of L1.28 RFLPs has been analysed in these families. Close linkage has been demonstrated between XLRP and L1.28 with a maximum lod score of 7.60 at a recombination fraction of 0.03 . This probe is potentially useful for counselling of XLRP families; however, more families need to be examined to exclude genetic heterogeneity.

Amniotic fluid alkaline phosphatase isoenzymes in the early prenatal diagnosis of cystic fibrosis

DAVID J H BROCK

Human Genetics Unit, University of Edinburgh, Western General Hospital, Edinburgh EH4 $2 X Y$.

Second trimester amniotic fluid contains two major alkaline phosphatase (ALP) isoenzymes, one susceptible to inhibition by phenylalanine and the other to homoarginine. The proportions of these isoenzymes are constant between 15 and 21 weeks of gestation. In pregnancies where the fetus has cystic fibrosis there is a profound deficiency of the phenylalanine inhibitable form, thought to derive mainly from mucosal cells of the $\frac{\mathbb{D}}{\mathbb{D}}$ fetal intestine. Using both phenylalanine and homo- : arginine to define ALP isoenzymes, it has been possible $\overrightarrow{\vec{F}}$ to detect nine of ten cases of cystic fibrosis on stored amniotic fluids, while only nine of 831 control fluids were scored as false positives. Use of this system in pregnancies $\frac{\sigma}{\bar{n}}$ where there is an a priori risk of $1: 3$ (one or more previous $\frac{\rho}{\partial}$ affected children) would alter the odds on being affected $₫$ to $28: 1$. It is argued that this provides for the first time a satisfactory method for the early prenatal diagnosis of $\mathrm{C}$ cystic fibrosis.

Periconceptional vitamin supplementation and the prevention of neural tube defects in Northern Ireland and south-ce east England

MARY J SELLER* AND N C NEVIN $\dagger$

*Paediatric Research Unit, The Prince Philip Research Laboratories, Guy's Hospital Medical School, London; $\vec{\omega}_{\infty}$ and $\dagger$ Department of Medical Genetics, Institute of 으 Clinical Science, Belfast.

Periconceptional vitamin supplementation has been $\frac{7}{0}$ given to women at risk for neural tube defects (NTD) in Northern Ireland and south-east England since 1976, $\vec{\theta}$ first, until 1980, as part of the Multicentre Study, the results of which have been published (Smithells et al. Lancet 1980;i:339-40. Smithells et al. Lancet 1983;i: 1027-31), and subsequently on an individual basis. The two areas represent the extremes of birth prevalence of $\bar{\partial}$ NTD within the United Kingdom. We have compared the findings on roughly 200 fully supplemented preg- $\mathbb{\Phi}$ nancies from each. The results show that the beneficial effect of prevention of NTD is more marked in Northern $\overrightarrow{\bar{D}}$ Ireland, which has a high birth prevalence, than in 3 south-east England, which has a low prevalence. This has important implications for any future large scale trials or use of this form of therapy.

Cellular hypersensitivity to UV-A in fibroblasts from actinic reticuloid

P K BOTCHERBY*, I A MAGNUS $\dagger$, B MARIMO*, AND F GIANNELLI*

*Paediatric Research Unit, Guy's Hospital; and †Depart-음 ment of Photobiology, Institute of Dermatology, London. I

Actinic reticuloid (AR), a severe photodermatosis, characterised by skin sensitivity to UV-B, UV-A, and part of the visible spectrum, and by dense dermal in- $N$ filtrates resembling those of mycosis fungoides, shows inN vitro cellular hypersensitivity to UV-A. This is the first ${ }_{\sigma}^{\omega}$ reported human disease exhibiting such cellular sensitivity. We have shown that fibroblasts from AR patients following irradiation with UV-A (320 to $400 \mathrm{~nm})$ exhibit $\bar{\complement}$ persistent inhibition of RNA synthesis and marked? cytopathic changes which are not observed in cells from $\square$ patients with Bloom syndrome or xeroderma pigmento-O sum. Furthermore, the AR cells have more DNA single strand breaks than normal cells when irradiated with $\mathbb{D}^{+}$ UV-A at temperatures compatible with enzyme activity. 응 Germicidal UVR (c 95\% $254 \mathrm{~nm}$ ) stimulates DNA 
repair synthesis and inhibits DNA replication to a normal extent in the AR cells. We advanced the hypothesis that inefficient neutralisation of free radicals might explain the cellular phenotype of $A R$ and could contribute to the establishment of a vicious circle that would favour the chronic clinical course and persistent lymphohistiocytic skin infiltrates characteristic of the disease.

Application of DNA probes from the gene of coagulation factor IX to the study of Christmas disease

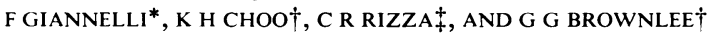
*Paediatric Research Unit, Guy's Hospital Medical School, London; †Sir William Dunn School of Pathology, University of Oxford; and $\ddagger$ Haemophilia Centre, Churchill Hospital, Oxford.

Christmas disease is an $\mathrm{X}$ linked recessive haemorrhagic disorder due to deficiency of coagulation factor IX. The gene for this factor has been cloned and probes derived from the gene have been used to analyse patients with factor IX deficiency. Among the many clinical and laboratory subtypes of Christmas disease, patients who develop antibodies against exogenous factor IX are the most interesting and pose the greatest clinical challenge. We have found that gross gene deletions are the principal cause of immunological intolerance to factor IX in patients with Christmas disease. DNA studies are useful in the genetic counselling of families segregating for deletions of the factor IX gene, while intragenic restriction fragment length polymorphisms should extend the usefulness of DNA analysis to most families segregating for Christmas disease. One such polymorphism with ideal frequency in Caucasians has been identified, its position within the gene has been determined, and its usefulness in genetic counselling demonstrated.

The application of restriction fragment length polymorphisms to genetic counselling

J F CLAYTON, S S BHATTACHARYA, A F WRIGHT, AND W H PRICE

MRC Clinical and Population Cytogenetics Unit, Western General Hospital, Edinburgh.

The increasing use of restriction fragment length polymorphisms (RFLP) in the localisation of disease loci, using linkage analysis, requires that there is optimal use of the available information for the purposes of genetic counselling. It is usual practice to define the recombination fraction (theta) between a probe and disease locus as that which has the maximum likelihood and the risk to a.person is calculated from this figure. An alternative approach is to derive a probability distribution of theta from the data and to use this to calculate the mean risk for a person. This value (obtained by integrating the risk at given theta biased by the probability distribution for that value of theta over all theta) is the value for which the expected error is a minimum. In the hypothetical case of an X linked disease and RFLP for which 25 informative meiotic events have been observed, one of which shows recombination, the most likely value of theta is 0.04 . The risk to a person of inheriting the disease from his doubly heterozygous mother (linkage phase known) would be put at $4 \%$ or $96 \%$ by the conventional method, depending on which allele he inherits. His mean risk, however, would be $8 \%$ or $92 \%$. In some situations, the difference between maximum likelihood and mean risk estimates could be clinically significant.

\section{Chromosome breaks and cancer sites in Fanconi anaemia} ANGELA I TAYLOR

Academic Department of Obstetrics and Gynaecology, Middlesex Hospital Medical School, London WI.

Orcein stained and Giemsa banded peripheral blood cultures from 15 patients homozygous or heterozygous for Fanconi anaemia were studied in order to ascertain whether chromosome breakage is random. The results were compared with those from 18 non-anaemic, nonleukaemic patients referred for chromosome study. Fifty-seven breakage sites were identified in the Fanconi patients and 18 in the controls. Breakage sites were defined as the sites of simple breaks or of structural rearrangements. Sites in Fanconi patients and controls were compared with 174 sites in leukaemia and lymphoma patients (data mainly from published reports). Breakage patterns are similar in all three groups and in all breakage is non-random with chromosomes 16 and $X$ having a deficiency of breaks. Certain sites are particularly prone to breakage with $22 \mathrm{q} 11,9 \mathrm{q} 34,14 \mathrm{q} 32,6 \mathrm{q} 21,6 \mathrm{q} 25,8 \mathrm{q} 22$, $21 \mathrm{q} 22,7 \mathrm{q} 22,15 \mathrm{q} 26,17 \mathrm{q} 22,18 \mathrm{q} 21,9 \mathrm{q} 22,11 \mathrm{q} 13,1 \mathrm{q} 22$, and $3 q 21$ the most frequent. Of the 57 sites identified in Fanconi anaemia, 37 were also seen in leukaemia and lymphoma patients and two are at the sites of oncogenes: 6q24-25 C-myb, and 15q25-26 C-fes (Harper et al. Nature 1983;304:169). There is also overlap between Fanconi breaks and those in translocations of solid tumours: $\mathrm{Ca}$ bronchus, 5p11, 2q21, and 11q13 (Pickthall VJ. Br J Cancer 1976;34:272), Ca ovary, 6q21 (Wake et al. Cancer Genet Cytogenet 1980;2:87), and retinoblastoma, 13q14 (Cavanee et al. Nature 1983;305:779). Comparison of the 57 Fanconi breaks with the nine established inherited fragile sites reveals that only $11 \mathrm{q} 13$ (the most frequent break in the present Fanconi series) is present in both groups.

\section{Karyotype abnormalities in non-Hodgkin lymphomas} K L GAUNT

Cytogenetics Department, Royal Victoria Infirmary, Newcastle upon Tyne.

Karyotype abnormalities were found in nine out of 13 non-Hodgkin lymphomas. The observed non-random involvement of chromosomes 1, 6, 9, and 14 was nonspecific in that it was associated with chromosome breakpoints rather than recurrent marker chromosomes. The recurrent markers which were found were similar to those of previously published studies and these are generally associated with the lymphoproliferative disorders $(14 q+, 6 q-, 18 q-)$. Three systems of classification were used in an attempt to correlate histological classification and chromosome anomalies. Limited 
histological associations were found in an analysis of 72 published cases. The frequency of abnormalities of chromosomes 14 and 18 showed the largest disparity between disease types.

Regional chromosomal localisation of the $\mathrm{N}$-ras oncogene to 1 cen $\rightarrow$ p21

$M$ DAVIS AND $S$ MALCOLM

MRC Human Genetic Diseases Research Group, Biochemistry Department, Queen Elizabeth College, University of London, London W8 $7 \mathrm{AH}$.

Karyotypic abnormalities are a feature of human tumours. The non-random nature of these changes has become evident since consistent chromosome aberrations are associated with certain types of malignancy. Chromosome abnormality may precede malignancy, suggesting that genomic rearrangement or imbalance has a primary role to play in the development of malignancy. There is concordance between the chromosomal position of oncogenes and the breakpoints involved in chromosomal translocations and deletions in various forms of human cancer. The ras family of oncogenes appears to be associated with solid tumours. The Harvey-ras oncogene has been assigned to the short arm of chromosome 11, a region where deletions occur in the aniridia-Wilms' tumour syndrome and in human melanomas. The Kirsten-ras oncogene, assigned to chromosome 12, is the most commonly activated oncogene in experiments using solid tumour material and cell lines from colon carcinomas. Recently a third member of the ras family, N-ras, was isolated and shown to be active in sarcoma and neuroblastoma cell lines. This gene was assigned to chromosome 1 using somatic cell hybrids. Using in situ hybridisation, we have confirmed this assignment and localised the gene to the region 1 cen $\rightarrow$ p21. The nonrandom involvement of chromosome 1 has been observed repeatedly in a variety of neoplasms, both of haematological origin and in solid tumours. The observed aberrations include translocations, trisomies, and deletions. It is as yet unknown whether these chromosomal alterations have a role to play in the activation of $\mathrm{N}$-ras in human tumours.

Incidence of autosomal trisomies at amniocentesis: lower than predicted frequencies in older mothers

J R W YATES AND M A FERGUSON-SMITH

Duncan Guthrie Institute of Medical Genetics, University of Glasgow, Yorkhill, Glasgow G3 8SJ.

Thanks to the kind collaboration of 58 centres in the United Kingdom, Europe, Canada, and South Africa, the age of the mother at amniocentesis and the results of fetal chromosome analysis have been collected in $\mathbf{5 2} 965$ pregnancies tested because maternal age was 35 years or above (Ferguson-Smith and Yates, Prenatal Diagnosis, in preparation). A total of 773 autosomal trisomies were observed. For trisomy 21 the incidence rose exponentially up to a maternal age of 46 years. In the 129 pregnancies in mothers aged 47 years and above there were three cases of trisomy 21 compared to 11.5 predicted by logistic regression analysis on the assumption that the incidence continues to rise exponentially at the upper end $\stackrel{\Phi}{\stackrel{\Phi}{D}}$ of the age range. Assuming a Poisson distribution, the probability of observing at most three cases when $11.5 \stackrel{\mathrm{O}}{\mathrm{O}}$ are expected is 0.003 . Trisomy 18 showed the same pattern. The incidence rose up to the age of 43 years, but $\frac{\mathrm{C}}{0}$ in the 1884 pregnancies in mothers over this age there $\frac{\bar{c}}{\mathrm{D}}$ were nine cases of trisomy 18 compared to $28 \cdot 3$ predicted $\vec{\nabla}$ (probability $=0.00002$ ). With only 39 cases of trisomy $13 \AA$ the analysis is less reliable, but the pattern appeared to be similar with a rising incidence up to the age of 42 years and a deficiency of cases in the 3796 pregnancies in older. women (number expected $=15 \cdot 9$, number observed $=2, \overrightarrow{\vec{\omega}}$ probability $=0 \cdot 00002$ ). The lower than predicted incidence $\omega_{\sigma}$ of autosomal trisomies in older mothers may reflect a corresponding trend in the frequency of trisomic conceptions. Alternatively, it could be the result of increasing is fetal loss of such conceptions before the time of amnio- $\vec{i}$ centesis in older women. Evidence in favour of the second of these possibilities was discussed.

The use of chorionic villi for early prenatal diagnosis of metabolic disorders

A H FENSOM*, M JACKSON*, N SANGUINETTI*, C H RODECK $\dagger, \vec{\bullet}$ J M MORSMAN $\dagger$, D V COLEMAN $\ddagger$, AND D HEATON $\ddagger$

*Paediatric Research Unit, Guy's Hospital Medical School; †Department of Obstetrics and Gynaecology, King's College Hospital; and $\ddagger$ Cytogenetics Unit, St Mary's Hospital Medical School, London.

A study was made of the activities of eight lysosomal and $\stackrel{\varnothing}{\varnothing}$ one cytosolic enzyme in cultured and uncultured speci- $\stackrel{2}{\Rightarrow}$ mens of chorionic villi obtained in two centres $(\mathrm{KCH}$ and SMHMS) from women undergoing termination of pregnancy. Activity of $\alpha$-iduronidase was low in uncultured villi but increased on culture to levels comparable to those in amniotic cells. Cultured villi may be the material of choice for prenatal diagnosis of Hurler's 0 disease. In contrast, ceramidase and $\alpha$-fucosidase were $\dot{0}$ more active in uncultured villi. Further, ceramidase was $\underset{3}{3}$. markedly more active in villi than in cultured amniotic cells, indicating an advantage of direct assay of villi for prenatal diagnosis of Farber's disease. Mean activities of $\mathrm{O}$ $\beta$-galactosidase, $\beta$-glucosidase, arylsulphatase $\mathrm{A}$, and total hexosaminidase were higher in cultured than in $\frac{D}{O}$ uncultured villi, but the effect was less marked than for $\alpha$-iduronidase. $\alpha$-glucosidase and hexosaminidase $A$ had $N$ similar activities in the two materials. Activity of cys- or tathionine $\beta$-synthase was barely detectable in villi, but $\tilde{O}$ increased on culture to levels comparable to those in $\underset{\mathrm{N}}{ }$ amniotic cells, demonstrating the necessity for culture for prenatal diagnosis of homocystinuria. When a pregnancy at risk for metachromatic leucodystrophy was $\stackrel{0}{\simeq}$ monitored by assay of arylsulphatase $A$ in villi and $\mathbb{Q}$ cultured villi, a heterozygous fetus was predicted, $\stackrel{+}{?}$ whereas at amniocentesis the fetus was found to be $\underset{T}{0}$ affected. The result with the cultured villi was explained by maternal contamination but this was not proven for $\stackrel{\mathbb{\Omega}}{\Omega}$ the uncultured villi. The case illustrates the pitfalls that $\mathbb{D}$ can still be associated with this method of prenatal diagnosis. 
The use of chorionic villus biopsy for first trimester prenatal diagnosis of chromosome defects

D E HEATON, B H CZEPULKOWSKI, D H HORWELL, AND D V COLEMAN

St Mary's Hospital, London W2.

We have developed a technique for the culture of chorionic villi for chromosome analysis which is simple, reliable, and suitable for use in any routine diagnostic laboratory. This method combines the use of dissection of villous from non-villous material with the exposure of the mesenchyme core by thorough maceration, thus eliminating the need for trypsinisation and filtration procedures used in previous techniques. Using this technique, we have cultured chorionic villi aspirated from first trimester pregnancies which were to be terminated for medical reasons, in parallel with fetal material obtained after termination. Karyotypes were obtained from villous cultures in over $80 \%$ of cases, between 14 and 24 days from the sample being taken, and with an accurate diagnosis in over $90 \%$ of cases. In addition, we have recently diagnosed trisomy 13 in a first trimester pregnancy of a 41 year old woman who had requested prenatal diagnosis. We also reported on preliminary experiments with a direct method for obtaining metaphase spreads from spontaneous mitoses in the cytotrophoblast layer, and compared the results with those obtained by culturing. 